\title{
Hyperfine-mediated transitions between a Zeeman split doublet in GaAs quantum dots: The role of the internal field
}

\author{
Sigurdur I. Erlingsson, and Yuli V. Nazarov \\ Delft University of Technology, Department of Applied Physics, Lorentzweg 1, 2628 CJ Delft, The Netherlands
}

(Received 12 February 2002; published 29 October 2002)

\begin{abstract}
We consider the hyperfine-mediated transition rate between Zeeman split states of the lowest orbital level in a GaAs quantum dot. We separate the hyperfine Hamiltonian into a part which is diagonal in the orbital states and another one which mixes different orbitals. The diagonal part gives rise to an effective (internal) magnetic field which, in addition to an external magnetic field, determines the Zeeman splitting. Spin-flip transitions in the dots are induced by the orbital-mixing part accompanied by an emission of a phonon. We evaluate the rate for different regimes of applied magnetic field and temperature. The rates we find are bigger than the spinorbit-related rates provided the external magnetic field is sufficiently low.
\end{abstract}

DOI: 10.1103/PhysRevB.66.155327

PACS number(s): 73.21.La, 71.70.Jp

\section{INTRODUCTION}

Manipulation of an individual quantum state in a solidstate system is currently the focus of an intense research effort. There are various schemes which have been proposed and they are in various stages of development. ${ }^{1-4}$ Many proposals concentrate on the spin degree of freedom of an electron in a quantum dot. Recent experiments indicate very long spin decoherence times and small transition rates between states of different $\operatorname{spin}^{5-7}$ in some semiconductor heterostructures.

A characteristic feature of a quantum dot is its discrete energy spectrum. Depending on the strength of the confinement, both potential and magnetic, an orbital level energy separation of a few $\mathrm{meV}$ is possible. ${ }^{8}$ This large energy separation strongly affects inelastic transition rates in the dot. The nuclei in GaAs have a substantial hyperfine interactions with the conduction electrons. This makes it relevant to investigate hyperfine-related effects in quantum dots in GaAs$\mathrm{AlGaAs}$ heterostructures.

Manipulating the electron spin while maintaining phase coherence requires that it should be relatively well isolated from the environment. Coupling a quantum dot, or any closed quantum system, to its environment can cause decoherence and dissipation. One of the measures of the strength of the coupling to the environment are the transition rates, or inverse lifetimes, between the quantum dot states. In GaAs there are two main mechanisms that can cause finite lifetimes of the spin states. These are the spin-orbit interaction and the hyperfine interaction with the surrounding nuclei. If a magnetic field is applied the change in Zeeman energy due to a spin flip has to be accompanied by phonon emission. For two electron quantum dots, where the transitions are between triplet and singlet spin states, both spin-orbit ${ }^{9,10}$ and hyperfine ${ }^{11}$-mediated transitions have been studied. In both cases the transition rates are much smaller than the usual phonon rates, the spin-orbit rate being higher except when the excited singlet and triplet states cross. ${ }^{11}$ For low magnetic fields, i.e., away from the singlet-triplet transition, the energy of the emitted phonon can be quite large and the transitions involve deformation phonons rather than piezoelectric ones.
If there are an odd number of electrons in the dot the ground state is usually a spin doublet so that energy change associated with the spin flip is the electron Zeeman energy. Owing to the small $g$ factor in GaAs this energy is rather small compared to the orbital level spacing, and the dominating phonon mechanism is due to piezoelectric phonons. Recently spin-orbit-mediated spin-flip transitions between Zeeman levels were investigated ${ }^{12}$. Due to Kramer's degeneracy the transition amplitude for the spin flip is proportional to the Zeeman splitting. This results in a spin-orbit spin-flip rate proportional to the fifth power of the Zeeman splitting.

In this paper we consider hyperfine-mediated transitions between Zeeman split levels in a quantum dot. The transition amplitude remains finite at zero external magnetic field, resulting in a spin-flip rate [Eq. (27)] that is proportional to the cube of the Zeeman splitting. The cause of this is an internal magnetic field due to the hyperfine interaction. We consider the important concept of internal magnetic field in some detail. Since the parameters of hyperfine interaction between conduction-band electrons and underlying nuclei in GaAs have been extensively investigated, ${ }^{13,14}$ including the Overhauser effect and spin relaxation in GaAs/AlGaAs heterostructures, ${ }^{15-19}$ we are able to calculate the hyperfinemediated spin-flip transition rate for quantum dots in such structures.

Upon completion of this work we learned about recent results of Khaetskii, Loss, and Glazman. ${ }^{20}$ They consider essentially the same situation and model and obtain electronspin decoherence without considering any mechanism of dissipation. This is in clear distinction from the present result for the spin-flip rate that requires a mechanism of dissipation, i.e., phonons.

The rest of the paper is organized as follows: in Sec. II the model used is introduced in addition to the basic assumptions and approximations used, Sec. III deals with the internal magnetic field due to the hyperfine interaction, and Sec. IV contains the derivation of the transition rates. Finally, in Sec. $\mathrm{V}$ the results are discussed.

\section{MODEL AND ASSUMPTIONS}

We consider a quantum dot embedded in a $\mathrm{AlGaAs} / \mathrm{GaAs}$ heterostructure. Since the details of the quantum dot eigen- 
states are not important for now, it suffices to say that the energy spectrum is discrete and the wave functions are localized in space. The spatial extension of the wave function in the lateral and transverse directions (growth direction) are denoted with $l$ and $z_{0}$, respectively. Quantum dots in these heterostructures are formed at a GaAs/AlGaAs interface, where the confining potential is very strong so that $l \gg z_{0}$. We define the volume occupied by an electron as $V_{\mathrm{QD}}=\pi l^{2} z_{0}$. The Hamiltonian of the quantum dot can be written in the form

$$
H_{0}=\sum_{l} \epsilon_{l}+g \mu_{B} \mathbf{B} \cdot \hat{\mathbf{S}}|l\rangle\langle l|,
$$

where $\varepsilon_{l}$ are the eigenenergies which depend on the structure of the confining potential and the applied magnetic field $\mathbf{B}$. The magnetic field also couples to the electron spin via the Zeeman term, where $g$ is the conduction-band $g$ factor and $\mu_{B}$ is the Bohr magneton.

Since the $\Gamma$ point of the conduction band in GaAs is mainly composed of $s$ orbitals the dipole interaction with the nuclei vanishes and the hyperfine interaction can be described by the usual contact term

$$
H_{\mathrm{HF}}=A \hat{\mathbf{S}} \cdot \sum_{k} \hat{\mathbf{I}}_{k} \delta\left(\mathbf{r}-\mathbf{R}_{k}\right),
$$

where $\hat{\mathbf{S}}\left(\hat{\mathbf{I}}_{k}\right)$ and $\mathbf{r}\left(\mathbf{R}_{k}\right)$ denote, respectively, the spin and position of the electron ( $k$ th nuclei). The delta function indicates that the pointlike nature of the contact interaction will result in a position-dependent coupling. The coupling constant $A$ has the dimension volume $\times$ energy. To get a notion of the related energy scale, it is straightforward to relate $A$ to the energy splitting of the doublet for a fully polarized nuclear system,

$$
E_{n}=A C_{n} I,
$$

$C_{n}$ being density of nuclei and $I$ the spin of a nucleus. In GaAs this energy is $E_{n} \approx 0.135 \mathrm{meV}$, which corresponds to a magnetic field of about $5 \mathrm{~T}^{15}$ For a given quantum dot geometry the number of nuclei occupying the dot is defined as $N_{\mathrm{QD}}=C_{n} V_{\mathrm{QD}}$. Since the Ga and As nuclei have the same spin and their coupling constants are comparable, we will assume that all the nuclear sites are characterized by the same hyperfine coupling $A$, and $C_{n} \approx a_{0}^{-3}$ where $a_{0}$ is the lattice spacing. For realistic quantum dots $N_{\mathrm{QD}} \approx 10^{4}-10^{6}$, and it is therefore an important big parameter in the problem.

The coupling between the electron and the phonon bath is represented with

$$
H_{\mathrm{ph}}=\sum_{\mathbf{q}, \nu} \alpha_{\nu}(\mathbf{q})\left(b_{\mathbf{q}, \nu} e^{i \mathbf{q} \cdot \mathbf{r}}+b_{\mathbf{q}, \nu}^{\dagger} e^{-i \mathbf{q} \cdot \mathbf{r}}\right),
$$

where $b_{\mathbf{q} \nu}^{\dagger}$ and $b_{\mathbf{q} \nu}$ are creation and annihilation operators for the phonon mode with wave vector $\mathbf{q}$ on branch $\nu$. In GaAs there are two different coupling mechanisms, deformation ones and piezoelectric ones. For transitions between Zeeman split levels in GaAs, i.e., low-energy emission, the most effective phonon mechanism is due to piezoelectric phonons.
We will assume that the heterostructure is grown in the [100] direction. This is the case for almost all dots and it imposes important symmetry relations on the coupling coefficient. The square of the coupling coefficient for the piezoelectric phonons is then given by (see Ref. 21)

$$
\alpha_{\nu}^{2}(\mathbf{q})=\frac{\left(e h_{14}\right)^{2} \hbar}{2 \rho c_{\nu} V q} A_{\nu}(\theta),
$$

where $\left(e h_{14}\right)$ is the piezoelectric coefficient, $\rho$ is the mass density, $c_{\nu}$ is the speed of sound of branch $\nu, V$ is normalization volume, we have defined $\mathbf{q}$ $=q(\cos \phi \sin \theta, \sin \phi \sin \theta, \cos \theta)$, and the $A_{\nu}$ 's are the socalled anisotropy functions, see Appendix B.

\section{INTERNAL MAGNETIC FIELD}

In this section we will introduce the concept of the effective magnetic field, the internal field, acting on the electron due to the hyperfine interaction. This internal field is a semiclassical approximation to the nuclear system, this approximation being valid in the limit of a large number of nuclei, $N_{\mathrm{QD}} \gg 1$. If the nuclei are noticeably polarized, this field coincides with the Overhauser field that represents the average nuclear polarization. It is important that the internal field persists even at zero polarization giving rise to Zeeman splitting of the order $E_{n} N_{\mathrm{QD}}^{-1 / 2}$.

First we write the Hamiltonian in Eq. (2) in the basis of the electron orbital states and present it as a sum of two terms

$$
H_{\mathrm{HF}}=H_{\mathrm{HF}}^{0}+V_{\mathrm{HF}},
$$

where the terms are defined as

$$
\begin{gathered}
H_{\mathrm{HF}}^{0}=A \sum_{l}|l\rangle\left\langle\left. l\left|\hat{\mathbf{S}} \cdot \sum_{k}\right|\left\langle\mathbf{R}_{k} \mid l\right\rangle\right|^{2} \hat{\mathbf{I}}_{k},\right. \\
V_{\mathrm{HF}}=A \sum_{l \neq l^{\prime}}|l\rangle\left\langle l^{\prime}\right| \hat{\mathbf{S}} \cdot \sum_{k}\left\langle l \mid \mathbf{R}_{k}\right\rangle\left\langle\mathbf{R}_{k} \mid l^{\prime}\right\rangle \hat{\mathbf{I}}_{k} .
\end{gathered}
$$

By definition $H_{\mathrm{HF}}^{0}$ does not couple different orbital levels. By combining Eqs. (1) and (7) one obtains the following Hamiltonian:

$$
H_{0}=\sum_{l}\left(\varepsilon_{l}+g \mu_{B} \hat{\boldsymbol{B}}_{l} \cdot \hat{\mathbf{S}}\right)|l\rangle\langle l| .
$$

We will regard the mixing term $V_{\mathrm{HF}}$ as a perturbation to $H_{0}$. The justification for this is that the typical fluctuations of the electron energy due to the hyperfine interaction are much smaller than the orbital energy separation.

We now concentrate on $H_{0}$ and formulate a semiclassical description of it. For this we consider the operator of the orbitally dependent effective magnetic field,

$$
\hat{\mathcal{B}}_{l}=\mathbf{B}+\frac{1}{g \mu_{B}} \hat{\mathbf{K}}_{l},
$$

where 


$$
\hat{\mathbf{K}}_{l}=\frac{E_{n}}{I C_{n}} \sum_{k}\left|\left\langle\mathbf{R}_{k} \mid l\right\rangle\right|^{2} \hat{\mathbf{I}}_{k} .
$$

Our goal is to replace the operator $\hat{\mathbf{K}}_{l}$ with a classical field. To prove the replacement is reasonable we calculate the average of the square for a given unpolarized nuclear state $|\boldsymbol{\mu}\rangle$,

$$
\mathbf{K}_{l}^{2}=\left\langle\boldsymbol{\mu}\left|\hat{\mathbf{K}}_{l}^{2}\right| \boldsymbol{\mu}\right\rangle \approx \frac{E_{n}^{2}}{N_{\mathrm{QD}}} .
$$

We cannot simply replace $\hat{\mathbf{K}}_{l}$ by its eigenvalues: as in the case of the usual spin algebra, different components of $\hat{\mathbf{K}}_{l}$ do not commute. In addition its square does not commute with individual components, $\left[\hat{\mathbf{K}}_{l}^{2}, \hat{K}_{l}^{\alpha}\right] \neq 0$. To estimate fluctuations of $\hat{\mathbf{K}}_{l}$ we calculate the uncertainty relations between its components,

$$
\Delta K_{l}^{\alpha} \Delta K_{l}^{\beta} \geqslant \frac{\alpha_{l} E_{n}^{2}}{N_{\mathrm{QD}}^{3 / 2}} \approx \mathbf{K}_{l}^{2} \frac{1}{N_{\mathrm{QD}}^{1 / 2}},
$$

where $\alpha_{l}$ is a numerical constant $o(1)$ which depends on the details of the orbital wave functions. Since $N_{\mathrm{QD}}^{-1 / 2} \ll 1$ we have proved that the quantum fluctuations in $\hat{\mathbf{K}}_{l}$ are much smaller than its typical amplitude. The semiclassical picture introduced above is only valid for high temperatures, $k T$ $\gg E_{n} N_{\mathrm{QD}}^{-1}$, where there are many states available to the nuclear system and the typical amplitude of $\mathbf{K}_{l}$ is proportional to $E_{n} N_{\mathrm{QD}}^{-1 / 2}$. For temperatures below $E_{n} N_{\mathrm{QD}}^{-1}$ the nuclear system will predominantly be in the ground state and the classical picture breaks down. This is similar to the quantum-mechanical description of a particle moving in a potential. At zero temperature it will be localized in some potential minimum and quantum mechanics will dominate. At sufficiently high temperatures the particle occupies higher-energy states and its motion is well described by classical mechanics. Having established this we can replace the average over the density matrix of the nuclei by the average over a classical field $\mathbf{K}_{l}$, and note that it has no "hat," whose values are Gaussian distributed,

$$
P\left(\mathbf{K}_{l}\right)=\left(\frac{1}{2 \pi \sigma_{l}^{2}}\right)^{3 / 2} \exp \left[-\frac{\left(\mathbf{K}_{l}-\mathbf{K}_{l}^{(0)}\right)^{2}}{2 \sigma_{l}^{2}}\right],
$$

where $\sigma_{l}^{2}=\frac{1}{3}\left(\left\langle\mathbf{K}_{l}^{2}\right\rangle-\left\langle\mathbf{K}_{l}\right\rangle^{2}\right)$ is the variance and $\mathbf{K}_{l}^{(0)}$ is the average, or Overhauser, field. In the case of a polarized nuclear system the variance decreases and the distribution becomes sharper around the Overhauser field, eventually becoming $P\left(\mathbf{K}_{l}\right)=\delta\left(\mathbf{K}_{l}-\mathbf{K}_{l}^{(0)}\right)$ as $\sigma_{l}^{2} \rightarrow 0$. The effective magnetic field acting on the electron is $\boldsymbol{B}_{l}=\mathcal{B}_{l} \mathbf{n}$ where $\mathbf{n}$ is the unit vector along the total field for a given $\mathbf{K}_{l}$, see Fig. 1 . For this configuration the spin eigenfunctions are $\left|\mathbf{n}_{ \pm}\right\rangle$, corresponding to eigenvalues

$$
\mathbf{n} \cdot \hat{\mathbf{S}}\left|\mathbf{n}_{ \pm}\right\rangle= \pm \frac{1}{2}\left|\mathbf{n}_{ \pm}\right\rangle .
$$

The effective Zeeman Hamiltonian is then

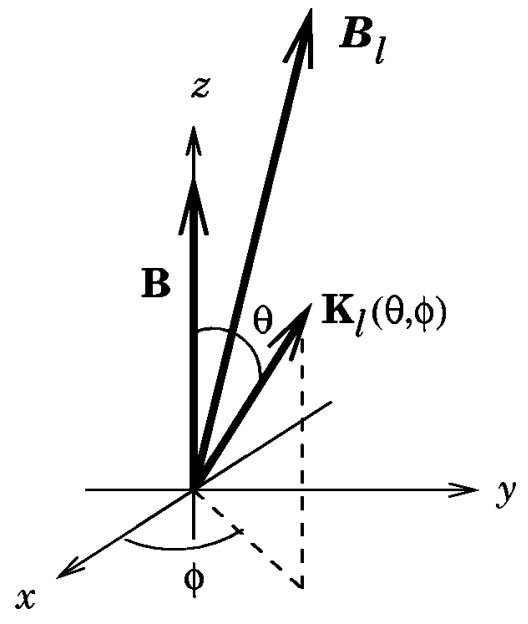

FIG. 1. The internal field coordinate system is set by the external magnetic field $\mathbf{B}$, i.e., $\mathbf{e}_{z} \| \mathbf{B}$. The combination of the external field and the internal field $\mathbf{K}_{l}$ results in an effective field $\mathcal{B}_{l}$.

$$
H_{\mathrm{Z}}=g \mu_{B} \boldsymbol{B}_{l} \cdot \hat{\mathbf{S}}
$$

in this given internal field configuration. The spectrum of $H_{0}$ thus consists of many doublets distinguished by the value of $\mathcal{B}_{l}$. The magnitude of the effective field $\mathcal{B}_{l}$ determines the Zeeman splitting of each doublet,

$$
\Delta_{l}=g \mu_{B} \mathcal{B}_{l}=\left(E_{B}^{2}+K_{l}^{2}+2 E_{B} K_{l} \cos \theta\right)^{1 / 2},
$$

where $E_{B}=g \mu_{B} B$ is the external field Zeeman energy.

We conclude this section with two remarks concerning time and energy scales. First, since the dynamics of $\mathbf{K}_{l}$ is due to the precession around the average electron spin, and the time scale for a full rotation is proportional to $N_{\mathrm{QD}} \hbar / E_{n}{ }^{22}$ For electron processes taking place on shorter time scales $\mathbf{K}_{l}$ plays the role of a "frozen disorder." At longer-time scales self-averaging over all values of $\mathbf{K}_{l}$ takes place. Second, the typical length of $\mathbf{K}_{l}$ is approximately $5 \times 10^{-4} \mathrm{meV}$ (for a value $N_{\mathrm{QD}} \approx 10^{5}$ ) which corresponds to a $100 \mathrm{G}$ magnetic field.

\section{TRANSITION RATE}

We concentrate on the transitions between the doublet components in Eq. (15). Assuming that the higher-energy doublet state is initially occupied, we will calculate the transition rate to the lower one. The transition must be accompanied by energy dissipation equal to $\Delta_{0}$. This energy cannot be absorbed by the nuclear system. ${ }^{11,23}$ So an external mechanism of energy dissipation is required. The most effective one in quantum dots is known to be phonons. However the phonons alone cannot change the electron spin so we need a mechanism which mixes spin and orbital degrees of freedom, that is, $V_{\mathrm{HF}}$ from Eq. (8). Thus the transition amplitude is proportional to both $V_{\mathrm{HF}}$ and the electron-phonon coupling $H_{\mathrm{ph}}$.

Here we assume that the electron is in the lowest orbital state $|0\rangle$ since the phonon mechanism will bring the electron to this state from any higher orbital on time scales much smaller than those related to transitions between the doublet 
components. Thus we consider an initial state of the entire system $|i\rangle=\left|0, \mathbf{n}_{-} ; \boldsymbol{\mu} ; \mathbf{N}\right\rangle$ which is a product state of the electron, nuclear $|\boldsymbol{\mu}\rangle$ and phonon $|\mathbf{N}\rangle$ systems, and the final state $|f\rangle=\left|0, \mathbf{n}_{+} ; \boldsymbol{\mu}^{\prime} ; \mathbf{N}^{\prime}\right\rangle$. Note that to a given state of the nuclear system $|\boldsymbol{\mu}\rangle$, which is a product state of all individual nuclei, there is an associated value of the classical field $\mathbf{K}_{l}$. The transition amplitude between $|i\rangle$ and $|f\rangle$, in second-order perturbation theory, reads

$$
\begin{aligned}
\mathcal{T}= & \sum_{l \neq 0}\left[\frac{\left\langle 0, \mathbf{n}_{+} ; \boldsymbol{\mu}^{\prime}\left|V_{\mathrm{HF}}\right| l, \mathbf{n}_{-} ; \boldsymbol{\mu}\right\rangle\left\langle l ; \mathbf{N}^{\prime}\left|H_{\mathrm{ph}}\right| 0 ; \mathbf{N}\right\rangle}{\left(\varepsilon_{0}-\varepsilon_{l}\right)+E_{B}}\right. \\
& \left.+\frac{\left\langle 0 ; \mathbf{N}^{\prime}\left|H_{\mathrm{ph}}\right| l ; \mathbf{N}\right\rangle\left\langle l, \mathbf{n}_{+} ; \boldsymbol{\mu}^{\prime}\left|V_{\mathrm{HF}}\right| 0, \mathbf{n}_{-} ; \boldsymbol{\mu}\right\rangle}{\left(\varepsilon_{0}-\varepsilon_{l}\right)-E_{B}}\right] .
\end{aligned}
$$

The summation is over virtual states involving higher orbitals, and the denominators in Eq. (18) contains the energy differences between different orbital states. The internal field depends on the orbital state, resulting in a rather complicated expression. Albeit the energy related to the internal field is much smaller than the orbital separation, we can safely replace the Zeeman splitting with $E_{B}$. The reason for that is that only at high external fields where $\Delta_{0} \approx E_{B}$ will the effects of the Zeeman splitting be appreciable in the denominator. The internal field also appears in the phonon rate since it determines the electron energy difference between the initial and final states. Since $H_{\mathrm{ph}}$ does not connect different nuclear states and conversely $V_{\mathrm{HF}}$ does not mix different phonon states the sums over intermediate phonon and nuclear states reduce to a single term. From this transition amplitude the transition rate is obtained via Fermis' golden rule,

$$
\Gamma_{\mathrm{sf}}=\frac{2 \pi}{\hbar} \sum_{\mathbf{N}^{\prime}} \sum_{\boldsymbol{\mu}^{\prime}} \mid \mathcal{T}^{2} \delta\left(E_{i}-E_{f}\right),
$$

where $E_{i}-E_{f}$ is the energy difference between the initial and final states of the combined systems. Substituting Eq. (18) into Eq. (19) we get the following relation for the spin-flip rate:

$$
\begin{aligned}
\Gamma_{\mathrm{sf}}= & \sum_{l, l^{\prime} \neq 0}\left\{\frac{\left\langle l, \mathbf{n}_{-} ; \boldsymbol{\mu}\left|V_{\mathrm{HF}}\right| 0, \mathbf{n}_{+}\right\rangle\left\langle 0, \mathbf{n}_{+}\left|V_{\mathrm{HF}}\right| l^{\prime}, \mathbf{n}_{-} ; \boldsymbol{\mu}\right\rangle}{\left[\left(\varepsilon_{0}-\varepsilon_{l}\right)+E_{B}\right]\left[\left(\varepsilon_{0}-\varepsilon_{l^{\prime}}\right)+E_{B}\right]}\right. \\
& \times \frac{2 \pi}{\hbar} \sum_{\mathbf{N}^{\prime}}\left\langle 0 ; \mathbf{N}\left|H_{\mathrm{ph}}\right| l ; \mathbf{N}^{\prime}\right\rangle\left\langle l^{\prime} ; \mathbf{N}^{\prime}\left|H_{\mathrm{ph}}\right| 0 ; \mathbf{N}\right\rangle \delta\left(E_{i}-E_{f}\right) \\
& +\frac{\left\langle l, \mathbf{n}_{-} ; \boldsymbol{\mu}\left|V_{\mathrm{HF}}\right| 0, \mathbf{n}_{+}\right\rangle\left\langle l^{\prime}, \mathbf{n}_{+}\left|V_{\mathrm{HF}}\right| 0, \mathbf{n}_{-} ; \boldsymbol{\mu}\right\rangle}{\left[\left(\varepsilon_{0}-\varepsilon_{l}\right)+E_{B}\right]\left[\left(\varepsilon_{0}-\varepsilon_{l^{\prime}}\right)-E_{B}\right]} \frac{2 \pi}{\hbar} \\
& \times \sum_{\mathbf{N}^{\prime}}\left\langle 0 ; \mathbf{N}\left|H_{\mathrm{ph}}\right| l ; \mathbf{N}^{\prime}\right\rangle\left\langle 0 ; \mathbf{N}^{\prime}\left|H_{\mathrm{ph}}\right| l^{\prime} ; \mathbf{N}\right\rangle \delta\left(E_{i}-E_{f}\right) \\
& +\frac{\left\langle 0, \mathbf{n}_{-} ; \boldsymbol{\mu}\left|V_{\mathrm{HF}}\right| l, \mathbf{n}_{+}\right\rangle\left\langle 0, \mathbf{n}_{+}\left|V_{\mathrm{HF}}\right| l^{\prime}, \mathbf{n}_{-} ; \boldsymbol{\mu}\right\rangle}{\left[\left(\varepsilon_{0}-\varepsilon_{l}\right)-E_{B}\right]\left[\left(\varepsilon_{0}-\varepsilon_{l^{\prime}}\right)+E_{B}\right]} \frac{2 \pi}{\hbar} \\
& \times \sum_{\mathbf{N}^{\prime}}\left\langle l ; \mathbf{N}\left|H_{\mathrm{ph}}\right| 0 ; \mathbf{N}^{\prime}\right\rangle\left\langle l^{\prime} ; \mathbf{N}^{\prime}\left|H_{\mathrm{ph}}\right| 0 ; \mathbf{N}\right\rangle \delta\left(E_{i}-E_{f}\right)
\end{aligned}
$$

$$
\begin{aligned}
& \times \frac{\left\langle 0, \mathbf{n}_{-} ; \boldsymbol{\mu}\left|V_{\mathrm{HF}}\right| l, \mathbf{n}_{+}\right\rangle\left\langle l^{\prime}, \mathbf{n}_{+}\left|V_{\mathrm{HF}}\right| 0, \mathbf{n}_{-} ; \boldsymbol{\mu}\right\rangle}{\left[\left(\varepsilon_{0}-\varepsilon_{l}\right)-E_{B}\right]\left[\left(\varepsilon_{0}-\varepsilon_{l^{\prime}}\right)-E_{B}\right]} \frac{2 \pi}{\hbar} \\
& \left.\times \sum_{\mathbf{N}^{\prime}}\left\langle l ; \mathbf{N}\left|H_{\mathrm{ph}}\right| 0 ; \mathbf{N}^{\prime}\right\rangle\left\langle 0 ; \mathbf{N}^{\prime}\left|H_{\mathrm{ph}}\right| l^{\prime} ; \mathbf{N}\right\rangle \delta\left(E_{i}-E_{f}\right)\right\} .
\end{aligned}
$$

The spin-flip rate depends on the initial state of the nuclear system $|\boldsymbol{\mu}\rangle$. This poses the problem of how to deal with the nuclear state $|\boldsymbol{\mu}\rangle$, since we already demoted all the spin operators to a collective classical variable. A conceptually simple solution lies in the fact that when Eqs. (20) and (8) are considered together one sees that the rate is a sum over all pairs of nuclei in the system. Focusing on a given pair of nuclei $k$ and $k^{\prime}$, all the other nuclei are unchanged when the electron spin is "scattered" upon by this pair. By simply redefining the classical field such that it is composed of all nuclei except this given pair we can circumvent the problem. This procedure will not change our previous result regarding the properties of $\mathbf{K}_{l}$ and by defining $|\boldsymbol{\mu}\rangle=\left|\mu_{k}\right\rangle\left|\mu_{k^{\prime}}\right\rangle$ makes it straightforward to work with the nuclear states in Eq. (20).

Although the transition rate can be very slow, the typical duration of a transition event is set by energy uncertainty, $\hbar / \Delta_{0}$. This is much shorter than the typical time for nuclear system dynamics so that the nuclear system is frozen in a given value of $\mathbf{K}_{0}$ during the transition. In this case, taking an average over $\mathbf{K}_{0}$, using the probability distribution in Eq. (14), is not well motivated. For now we will postpone the averaging over the classical field. Expanding the energy denominators to second order in the Zeeman splitting and performing the thermal average over nuclei spin pairs (see Appendix A) we obtain the following equation for the transition rate:

$$
\begin{aligned}
\Gamma_{\mathrm{sf}}= & G_{\text {corr }}\left(\sum _ { l \neq 0 } \left\{\frac{2 a_{l l} \gamma_{l l}}{\delta \varepsilon_{l}^{2}}\left(1+3 \frac{E_{B}^{2}}{\delta \varepsilon_{l}^{2}}\right)+\frac{2 \Re\left\{\tilde{a}_{l l} \tilde{\gamma}_{l l}\right\}}{\delta \varepsilon_{l}^{2}}\right.\right. \\
& \left.\times\left(1+\frac{E_{B}^{2}}{\delta \varepsilon_{l}^{2}}\right)\right\}+\sum_{l<l^{\prime} \neq 0}\left\{\frac{4 \Re\left\{a_{l l^{\prime}} \gamma_{l l^{\prime}}\right\}}{\delta \varepsilon_{l} \delta \varepsilon_{l^{\prime}}}\right. \\
& \times\left[1+\left(\frac{\left(\delta \varepsilon_{l}+\delta \varepsilon_{l^{\prime}}\right)^{2}}{\delta \varepsilon_{l} \delta \varepsilon_{l^{\prime}}}-1\right) \frac{E_{B}^{2}}{\delta \varepsilon_{l} \delta \varepsilon_{l^{\prime}}}\right] \\
& \left.\left.+\frac{4 \Re\left\{\tilde{a}_{l l^{\prime}} \tilde{\gamma}_{l l^{\prime}}\right\}}{\delta \varepsilon_{l} \delta \varepsilon_{l^{\prime}}}\left[1+\left(\frac{\left(\delta \varepsilon_{l}-\delta \varepsilon_{l^{\prime}}\right)^{2}}{\delta \varepsilon_{l} \delta \varepsilon_{l^{\prime}}}+1\right) \frac{E_{B}^{2}}{\delta \varepsilon_{l} \delta \varepsilon_{l^{\prime}}}\right]\right\}\right),
\end{aligned}
$$

where $\delta \varepsilon_{l}=\varepsilon_{0}-\varepsilon_{l}$. The parameters $a_{l l^{\prime}}, \tilde{a}_{l l^{\prime}}$ are related to the $V_{\mathrm{HF}}$ matrix elements

$$
\begin{aligned}
& a_{l l^{\prime}}=A^{2} C_{n} \int d^{3} \mathbf{R}_{k} \Psi_{l}^{*}\left(\mathbf{R}_{k}\right)\left|\Psi_{0}\left(\mathbf{R}_{k}\right)\right|^{2} \Psi_{l^{\prime}}\left(\mathbf{R}_{k}\right), \\
& \tilde{a}_{l l^{\prime}}=A^{2} C_{n} \int d^{3} \mathbf{R}_{k} \Psi_{l}^{*}\left(\mathbf{R}_{k}\right) \Psi_{l^{\prime}}^{*}\left(\mathbf{R}_{k}\right) \Psi_{0}\left(\mathbf{R}_{k}\right)^{2},
\end{aligned}
$$


and $\gamma_{l l^{\prime}}, \tilde{\gamma}_{l l^{\prime}}$ are generalized phonon transition rates

$$
\begin{aligned}
& \gamma_{l l^{\prime}}=\frac{2 \pi}{\hbar} \sum_{\mathbf{q} \nu} \frac{\alpha_{\nu}^{2}(\mathbf{q})\left[e^{-i \mathbf{q} \cdot \mathbf{r}}\right]_{0, l}\left[e^{i \mathbf{q} \cdot \mathbf{r}}\right]_{l^{\prime}, 0}}{1-e^{-\beta \hbar \omega_{\mathbf{q}, \nu}}} \delta\left(\hbar \omega_{\mathbf{q} \nu}-\Delta_{0}\right), \\
& \tilde{\gamma}_{l l^{\prime}}=\frac{2 \pi}{\hbar} \sum_{\mathbf{q} \nu} \frac{\alpha_{\nu}^{2}(\mathbf{q})\left[e^{-i \mathbf{q} \cdot \mathbf{r}}\right]_{0, l}\left[e^{i \mathbf{q} \cdot \mathbf{r}}\right]_{0, l^{\prime}}}{1-e^{-\beta \hbar \omega_{\mathbf{q}, \nu}}} \delta\left(\hbar \omega_{\mathbf{q} \nu}-\Delta_{0}\right) .
\end{aligned}
$$

Here we have only included the emission process since we assume that the spin is initially in the higher-energy doublet state.

Until now we have considered a general quantum dot and the rate in Eq. (21) is valid for any quantum dot. To proceed further we will specify the confining potential to be parabolic in the lateral direction, and in the transverse one a triangular well potential is chosen. The wave function is $\langle\mathbf{r} \mid l\rangle$ $\equiv \chi_{0}(z) \psi_{n, M}(r, \theta)$ where $n, M$ denote the orbital and angular momentum quantum numbers, respectively, of the DarwinFock solution and $\chi_{0}(z)$ is the wave function in the transverse direction. The generic quantum number thus becomes $l=(n, M)$. The square of the lateral confining length is $l^{2}$ $=\hbar^{2} / m^{*} \hbar \Omega$, where $m^{*}$ is the electron effective mass and $\Omega=\left[\Omega_{0}^{2}+\left(\omega_{c} / 2\right)^{2}\right]^{1 / 2}$ is the effective confining frequency, with $\hbar \Omega_{0}$ being the confining energy and $\omega_{c}=e B / m^{*}$ the cyclotron frequency. What remains is to calculate the $a$ 's and $\gamma$ 's in Eqs. (22)-(25). The results of these calculations are presented Appendix B.

In principle, it is possible to obtain the rate for all parameter values but to make the discussion more transparent we will consider two regimes of applied magnetic field (i) $E_{B}$ $\approx E_{n} N_{\mathrm{QD}}^{-1 / 2}$ and (ii) $E_{B} \gg E_{n} N_{\mathrm{QD}}^{-1 / 2}$. In regime (i) both $\Delta_{0}$ $\ll \hbar c_{\nu} l^{-1}$ and $\Delta_{0} \ll k T$ (for experimentally relevant temperatures) and only the lowest-order terms in $\Delta_{0} / \hbar c_{\nu} l^{-1}$ need to be considered. In GaAs $\hbar c_{l} l^{-1}=3.3 l^{-1} \mathrm{~nm} \times \mathrm{meV}$ and $\hbar c_{t} l^{-1}=2.0 l^{-1} \mathrm{~nm} \times \mathrm{meV}$ for the longitudinal and transverse branches, respectively. In the other regime the applied field dominates and the internal field may be ignored, but no additional assumptions are made in this case. The resulting hyperfine-mediated spin-flip rates are

$$
\begin{aligned}
\Gamma_{\mathrm{HF}}= & 0.34 \frac{G_{\mathrm{corr}}}{I^{2}} \frac{E_{n}^{2}}{N_{\mathrm{QD}}(\hbar \Omega)^{2}} \frac{\left(e h_{14} l\right)^{2} k T}{8 \pi \rho c^{5} \hbar^{4}}\left(E_{B}^{2}+K_{0}^{2}\right. \\
& \left.+2 E_{B} K_{0} \cos \theta\right) \text { for } E_{B} \approx E_{n} N_{\mathrm{QD}}^{-1 / 2}, \\
\Gamma_{\mathrm{HF}}= & \frac{G_{\mathrm{corr}}}{I^{2}} \frac{E_{n}^{2}\left[n\left(E_{B}\right)+1\right]}{N_{\mathrm{QD}}(\hbar \Omega)^{2}} \frac{\left(e h_{14} l\right)^{2} E_{B}^{3}}{8 \pi \rho c^{5} \hbar^{4}}\left(C_{0}\left(E_{B}\right)\right. \\
& \left.+\left(\frac{E_{B}}{\hbar \Omega}\right)^{2} C_{2}\left(E_{B}\right)\right) \text { for } E_{B} \gg E_{n} N_{\mathrm{QD}}^{-1 / 2} .
\end{aligned}
$$

Note that the rates have different dependencies on the emitted energy $\Delta_{0}$. In Eq. (27) we introduce the functions $C_{0}$ and $C_{2}$ which contain the details of the higher orbitals and the anisotropy integrals. For low fields $\Delta_{0} \ll \hbar c_{\nu} l^{-1}$ these functions are constant. The saturation value of the spin-flip rate in

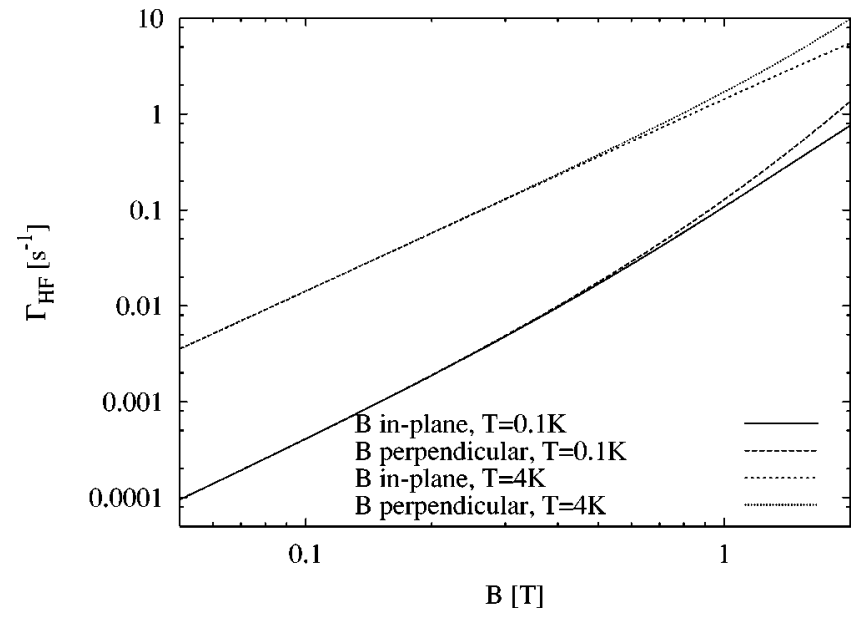

FIG. 2. The hyperfine-mediated spin-flip rate for a quantum dot with $z_{0}=10 \mathrm{~nm}$ and $\hbar \Omega_{0}=2 \mathrm{meV}$, plotted as a function of external magnetic field for two different temperatures $T=0.1 \mathrm{~K}$ and $4 \mathrm{~K}$.

Eq. (26), for some typical value of $K_{0}=5 \times 10^{-4} \mathrm{meV}$, is very low, $\Gamma_{\mathrm{HF}}<10^{-6} \mathrm{~s}$. This results in a lifetime of days, which will be extremely difficult to measure. For regime (ii) we have plotted the general spin-flip rate in Eq. (27) for different confining energies and temperatures in Figs. 2 and 3 , for both in-plane and perpendicular applied magnetic field. Due to the Bose distribution function factor $\left(n\left(E_{B}\right)+1\right)$, there is a crossover from $\Gamma_{\mathrm{HF}} \propto k T E_{B}^{2}$ to $\Gamma_{\mathrm{HF}} \propto E_{B}^{3}$ that occurs around $E_{B} \approx k T$. In both Figs. 2 and 3 this crossover is observed for the $T=0.1-\mathrm{K}$ curves around $B=0.34 \mathrm{~T}$. For the $T=1-\mathrm{K}$ curves the crossover occurs around $12 \mathrm{~T}$. In the case of the higher confining energy there is small difference between the in-plane and perpendicular directions of the external magnetic field. For the lower confining energy there is a substantial difference between the two directions of magnetic field. In this case the approximation $\Delta_{0} \ll \hbar c_{\nu} l^{-1}$ is no longer valid and the $\Delta_{0}$ dependence of the rate is changed by the $C$ functions. The values of the rates are quite small, depending

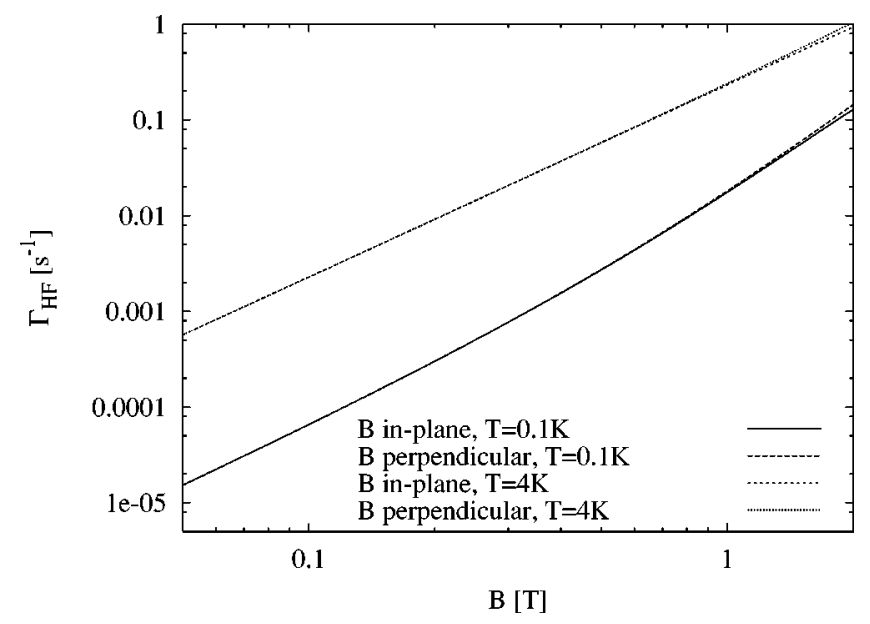

FIG. 3. The hyperfine-mediated spin-flip rate for a quantum dot with $z_{0}=10 \mathrm{~nm}$ and $\hbar \Omega_{0}=5 \mathrm{meV}$, plotted as a function of external magnetic field for two different temperatures $T=0.1 \mathrm{~K}$ and $4 \mathrm{~K}$. 
on the applied field, being $\sim 1 \mathrm{~s}^{-1}$ for $T=4 \mathrm{~K}$ at $B \approx 0.5 \mathrm{~T}$ for a confining frequency of $\hbar \Omega_{0}=2 \mathrm{meV}$.

\section{DISCUSSION}

Generally speaking, inelastic spin-flip rates require an external source of dissipation to facilitate the transitions. This is why at small Zeeman splittings they will contain a small factor reflecting the vanishing phonon density of states. For a spin-orbit rate, ${ }^{12}$ the Kramer's degeneracy results in this small factor being proportional to $E_{B}^{5}$. The presence of nuclear spins violates the Kramer's theorem. Thus, the hyperfine rate discussed in the present paper is proportional to $E_{B}^{3}$ and will dominate at sufficiently low fields.

Comparing the hyperfine rate in Eq. (27) to spin-orbitrelated rates ${ }^{12}$ and requiring that the rates are equal we obtain $\left(E_{B} / \hbar \Omega\right)^{2} \epsilon_{\beta}^{2} \approx E_{n}^{2} N_{\mathrm{QD}}^{-1}$, where $\epsilon_{\beta}$ determines the spin-orbit admixture strength. The extra factor $\left(E_{B} / \hbar \Omega\right)^{2}$ is due to Kramer's degeneracy which suppresses the spin-orbit rate compared to the hyperfine one at low fields. The crossover occurs at $E_{B} \approx \hbar \Omega\left(E_{n} N_{\mathrm{QD}}^{-1 / 2} / \epsilon_{\beta}\right)$, which correspond to magnetic field $B \approx 0.3 \mathrm{~T}$, assuming typical quantum dot parameters $z_{0}=10 \mathrm{~nm}$ and $\hbar \Omega_{0}=4 \mathrm{meV} .^{24}$

The role of the internal field produced by the nuclei is that the spin-flip rate does not vanish even in the absence of external magnetic field. We show that the minimum rate is rather small, corresponding to a relaxation time of the order of days. We believe that the internal field will play an important role when the full dynamics of the electron spin in the presence of the nuclear system is considered. Our model should also be applicable to other polar semiconductors which have nonzero nuclear spin, e.g., InAs, where the $g$ factor is much larger.

\section{ACKNOWLEDGMENTS}

One author (S.I.E.) would like to thank Daniela Pfannkuche and Alexander Chudnovskii for fruitful discussions. We also acknowledge discussions with Daniel Loss and Alexander V. Khaetskii. This work is a part of the research program of the "Stichting voor Fundementeel Onderzoek der Materie (FOM)."

\section{APPENDIX A: \\ THERMAL AVERAGE OVER NUCLEAR-SPIN PAIRS}

Even though we do not average over the classical field $\mathbf{K}_{l}$, which is fixed, there are still fluctuations in the nuclear system. Each pair of nuclei can fluctuate, without affecting the classical field. One should think of these fluctuations as small deviations around a given value of $\mathbf{K}_{l}$, i.e., we (thermally) average over the pairs for a fixed value of the classical field. When the thermal average over a pair of free nuclei is performed the following nuclear correlation function appears in Eq. (21):

$$
\begin{aligned}
G_{\text {corr }} & =\left(S_{+-}^{\alpha}\right)^{*} S_{+-}^{\beta}\left\langle\delta I_{k}^{\alpha} \delta I_{k^{\prime}}^{\beta}\right\rangle_{T} \\
& =\left(S_{+-}^{\alpha}\right)^{*} S_{+-}^{\beta}\left\langle\delta \hat{I}^{\alpha} \delta \hat{I}^{\beta}\right\rangle_{T} \delta_{k, k^{\prime}},
\end{aligned}
$$

where $\delta \hat{I}^{\alpha}=\hat{I}^{\alpha}-\left\langle I^{\alpha}\right\rangle_{T}$ and the electron-spin matrix elements are $S_{+-}^{\alpha}=\left\langle\mathbf{n}_{+}\left|S^{\alpha}\right| \mathbf{n}_{-}\right\rangle$. The Kronecker delta reflects that there are no correlations between two different nuclei and we have dropped the $k$ subscript in $\left\langle\delta \hat{I}^{\alpha} \delta \hat{I}^{\beta}\right\rangle_{T}$ since the nuclei is assumed to be identical. By defining the symmetric correlator

$$
g^{\alpha \beta}=\frac{1}{2}\left\langle\delta \hat{I}^{\alpha} \delta \hat{I}^{\beta}+\delta \hat{I}^{\beta} \delta \hat{I}^{\alpha}\right\rangle_{T},
$$

we get the following:

$$
G_{\mathrm{corr}}=\left(S_{+-}^{\alpha}\right)^{*} S_{+-}^{\beta}\left(g^{\alpha \beta}+i / 2 \epsilon^{\alpha \beta \gamma}\left\langle\hat{I}^{\gamma}\right\rangle_{T}\right)
$$

In an isotropic system, $\langle\hat{\mathbf{I}}\rangle_{T}=0$, the value of the correlation function is $G_{\text {corr }}=\frac{1}{2} \frac{1}{3} I(I+1)$.

\section{APPENDIX B: MATRIX ELEMENTS FOR A PARABOLIC QUANTUM DOT}

Using the Darwin-Fock solutions and the Fang-Howard variational solution for the triangular quantum well we obtain the following equation for Eqs. (22)-(25):

$$
\begin{aligned}
a_{l l^{\prime}} \gamma_{l l^{\prime}}= & \delta_{M, M^{\prime}} \frac{A^{2} C n}{V_{\mathrm{QD}} \zeta} \frac{\left(e h_{14} l\right)^{2} \Delta^{3}}{8 \pi \rho c^{5} \hbar^{4}}[n(\Delta)+1] \frac{\Gamma\left(n+n^{\prime}+|M|+1\right) 2^{-3\left(n+n^{\prime}+|M|\right)}}{n ! n^{\prime} !(n+|M|) !\left(n^{\prime}+|M|\right) !} \\
& \times\left\{\sum_{\nu} \frac{c^{5}}{c_{\nu}^{5}}\left(\frac{\Delta}{\hbar c_{\nu} l^{-1}}\right)^{2\left(n+n^{\prime}+|M|-1\right)} \int_{0}^{\pi} d(\cos \theta) A_{\nu}(\theta) \frac{(\sin \theta)^{2\left(n+n^{\prime}+|M|\right)} \exp \left[-\frac{1}{2}\left(\frac{\Delta \sin \theta}{\hbar c_{\nu} l^{-1}}\right]\right)}{\left[1+\left(\frac{\Delta \cos \theta}{3 \hbar c_{\nu} z_{0}^{-1}}\right)^{2}\right]^{3}}\right\},
\end{aligned}
$$


where $\zeta^{-1}=z_{0} \int d z|\chi(z)|^{4}$ and $c^{-5}=c_{l}^{-5}+c_{t}^{-5}$ is the effective sound velocity of the phonons. The anisotropy functions are

$$
A_{t}(\theta)=\frac{\sin ^{2} \theta\left(8 \cos ^{4} \theta+\sin ^{4} \theta\right)}{4},
$$

$$
A_{l}(\theta)=\frac{9 \cos ^{2} \theta \sin ^{4} \theta}{2} .
$$

The equation for $\tilde{a}_{l l^{\prime}} \tilde{\gamma}_{l l^{\prime}}$ is identical except for a different Kronecker delta function $\delta_{M,-M^{\prime}}$. The integral needs in general to be evaluated numerically but when $\Delta \ll \hbar c_{\nu} l^{-1}$ the exponential term and the denominator become unity and the resulting integral is simple to calculate.
${ }^{1}$ Y. Nakamura, Y. A. Pashkin, and J. S. Tsai, Nature (London) 398, 786 (1999).

${ }^{2}$ J. E. Mooij, T. P. Orlando, L. Levitov, L. Tian, and C. H. van der Wal, Science 285, 1036 (1999).

${ }^{3}$ B. E. Kane, Nature (London) 393, 133 (1998).

${ }^{4}$ D. Loss and D. P. DiVincenzo, Phys. Rev. A 57, 120 (1998).

${ }^{5}$ J. M. Kikkawa and D. D. Awschalom, Phys. Rev. Lett. 80, 4313 (1997).

${ }^{6}$ Y. Ohno, R. Terauchi, T. Adachi, F. Matsukura, and H. Ohno, Physica E 6, 817 (2000).

${ }^{7}$ T. Fujisawa, Y. Tokura, and Y. Hirayama, Phys. Rev. B 63, 081304(R) (2001).

${ }^{8}$ L. P. Kouwenhoven, C. M. Marcus, P. L. McEuen, S. Tarucha, R. M. Westervelt, and N. S. Windgreen, in Mesoscopic Electron Transport, Vol. 345 of NATO Advanced Studies Institute, Series E, edited by L. L. Sohn, L. K. Kouwenhoven, and G. Schön (Kluwer, Dordrecht, 1997).

${ }^{9}$ A. V. Khaetskii and Y. V. Nazarov, Phys. Rev. B 61, 12639 (2000).

${ }^{10}$ A. V. Khaetskii and Y. V. Nazarov, Physica E 6, 470 (2000).

${ }^{11}$ S. I. Erlingsson, Y. V. Nazarov, and V. I. Fal'ko, Phys. Rev. B 64, 195306 (2001).

${ }^{12}$ A. V. Khaetskii and Y. V. Nazarov, Phys. Rev. B 64, 125316 (2001).
${ }^{13}$ D. Paget, G. Lampel, B. Sapoval, and V. I. Safarov, Phys. Rev. B 15, 5780 (1977).

${ }^{14}$ D. Paget and V. L. Berkovits, Optical Orientation (NorthHolland, Amsterdam, 1984).

${ }^{15}$ M. Dobers, K. v. Klitzing, J. Schneider, G. Weimann, and K. Ploog, Phys. Rev. Lett. 61, 1650 (1988).

${ }^{16}$ A. Berg, M. Dobers, R. R. Gerhardts, and K. v. Klitzing, Phys. Rev. Lett. 64, 2563 (1990).

${ }^{17}$ I. D. Vagner, T. Maniv, and E. Ehrenfreund, Solid State Commun. 44, 635 (1982).

${ }^{18}$ S. E. Barrett, G. Dabbagh, L. N. Pfeiffer, K. W. West, and R. Tycko, Phys. Rev. Lett. 74, 5112 (1995).

${ }^{19}$ R. Tycko, S. E. Barret, G. Dabbagh, L. N. Pfeiffer, and K. W. West, Science 268, 1460 (1995).

${ }^{20}$ A. V. Khaetskii, D. Loss, and L. Glazman, Phys. Rev. Lett. 88, 186802 (2002).

${ }^{21}$ P. J. Price, Ann. Phys. (N.Y.) 133, 217 (1981).

${ }^{22}$ I. A. Merkulov, A. L. Efros, and M. Rosen, Phys. Rev. B 65, 205 309 (2002).

${ }^{23}$ J. H. Kim, I. D. Vagner, and L. Xing, Phys. Rev. B 49, 16777 (1994).

${ }^{24}$ Using the notation in Ref. 12 the spin-orbit admixture constant is $\epsilon_{\beta}=\beta \hbar / a$, where $a$ is the lateral quantum dot size, corresponding to $l$ in the present paper. 